\title{
Jan GolDSTEIN, The Post-Revolutionary Self. Politics and Psyche in France, 1750-1850
}

Jacques Guilhaumou

\section{(2) OpenEdition \\ Journals}

\section{Édition électronique}

URL : https://journals.openedition.org/ahrf/10252

DOI : $10.4000 /$ ahrf.10252

ISSN : 1952-403X

\section{Éditeur :}

Armand Colin, Société des études robespierristes

\section{Édition imprimée}

Date de publication : 15 mars 2006

Pagination : 220-221

ISSN : 0003-4436

\section{Référence électronique}

Jacques Guilhaumou, « Jan goldsteIn, The Post-Revolutionary Self. Politics and Psyche in France, 1750-1850", Annales historiques de la Révolution française [En ligne], 343 | janvier-mars 2006, mis en ligne le 17 novembre 2008, consulté le 23 avril 2022. URL : http://journals.openedition.org/ahrf/10252 ; DOI : https://doi.org/10.4000/ahrf.10252

Ce document a été généré automatiquement le 23 avril 2022.

Tous droits réservés 


\title{
Jan GoldSTEIN, The Post- Revolutionary Self. Politics and Psyche in France, 1750-1850
}

\author{
Jacques Guilhaumou
}

\section{RÉFÉRENCE}

Jan Goldstein, The Post-Revolutionary Self. Politics and Psyche in France, 1750-1850, Cambridge and London, Harvard University Press, 2005, 414 p., ISBN 0-674-01680-7,

\section{$41.5 €$}

1 Jan Goldstein est professeur d'histoire moderne de l'Europe à l'Université de Chicago. Dans le présent ouvrage, elle considère que nous pouvons apprendre beaucoup sur la culture politique d'une époque à partir du type de discours psychologique qu'elle adopte, en particulier sur le moi (self). Dès l'introduction, elle propose de distinguer d'une part le moi des Lumières fragmenté entre sa part inconsciente - son substratum et sa part consciente en dépit de la quête rousseauiste d'un moi unitaire, d'autre part le moi pris, avec les penseurs du XIX siècle, dans une répartition horizontale des rôles sous domination bourgeoise au titre d'une subjectivité jugée rationnelle et instaurée dans un univers essentiellement masculin.

2 De fait, les penseurs des Lumières, physiologues inclus à l'exemple de Bonnet, se heurtent, lorsqu'ils s'efforcent de promouvoir une politique de l'individualité (selfhood), au mécanisme souvent incontrôlable de l'imagination au sein de l'appareil mental. Le spectre d'un monde auto-référent, par le fait d'individus - qui plus est membres du peuple - perdant le contact avec la réalité des faits à cause de leur imagination débridée est présent dans les débats sur les réformes antérieures à 1789, en particulier autour des Édits de Turgot. Chez Condillac, le moi demeure ainsi fragmenté au titre de la difficulté à distinguer dans l'analyse les objets réels des objets imaginaires. 
3 Un fort intéressant chapitre sur «l'école révolutionnaire de l'imagination " précise alors dans quelle mesure, tant dans le domaine des fêtes qu'au sein de l'idéologie sensationnaliste de facture condillacienne fortement présente dans les nouvelles institutions savantes, les révolutionnaires s'efforcent de donner une direction rationnelle à l'imagination individuelle. Il s'agit alors de stabiliser le pouvoir de l'imagination au plus grand profit de la politique républicaine et de ses idéaux patriotiques. Ainsi l'idéal égalitaire permet à chaque individu de concrétiser dans son esprit par l'image une réalité en devenir, sans construire des fictions, ce qu'on appelle alors parfois des « châteaux en Espagne ».

4 Cependant la politique de l'individualité en reste encore à une vision passive et fragmentée du moi, même chez un Sieyès qui pousse très loin la réflexion sur l'activité du moi dans ses manuscrits. Mais il le fait par un effort intermittent, de fait très soutenu dans les années 1770, puis repris vingt ans plus tard pendant le Directoire pour aboutir à une systématisation dans les années 1810 qui nous situe déjà dans une autre conjoncture. Jan Goldstein resitue ainsi la métaphysique sieyèsienne du moi et de son activité, telle que nous l'avons décrite ailleurs, dans un contexte qui ne permet pas vraiment à Sieyès de construire de manière définitive le moi. La rencontre entre Sieyès et Maine de Biran, certes avérée par des faits ténus, marquerait alors le basculement dans un nouveau régime du moi formulé à diverses reprises dans le Journal de Maine de Biran des années 1814-1816.

5 Jan Goldstein consacre alors une grande part de son ouvrage à la formation du « moi bourgeois", principalement chez Victor Cousin, et plus largement chez les Doctrinaires, avec une attention particulière pour Guizot. Elles s'intéresse aussi à de nouveaux domaines de recherche, et tout particulièrement à la phrénologie définie comme « la seule vraie science de l'esprit» au plus haut de sa popularité au cours des années 1820-1840.

6 Il apparaît ainsi, dans le mouvement de la pensée devenue progressivement hégémonique de Victor Cousin, un moi justificateur de l'ordre bourgeois, un moi donné entier et a priori pour légitimer la domination du bourgeois mâle. L'unité dynamique du moi apparaît, dans les écrits de Victor Cousin, comme un moi actif, éloigné de l'anxiété issue de la vision passive du moi des sensualistes. Ainsi les penseurs des Lumières n'ont pas su faire pour eux-mêmes « un moi qui persiste » précise Victor Cousin. Cependant, ce «moi bourgeois », ce moi substance conçu à distance d'un moi défini à partir d'une collection de sensations demeure sous contrôle dans la mesure où il conjugue liberté individuelle et dépendance de manière à garantir le statu quo de l'ordre établi, de façon à faciliter le contrôle de classe.

7 Actuellement, le débat sur l'existence ou non d'une "classe bourgeoise ", au tournant des XVIII et XIXe siècles, revient à l'ordre du jour sous l'influence des travaux de Sarah Maza, en particulier de son livre récent sur The Myth of the French Bourgeoisie. An Essay on the Social Imaginary, 1750-1850. Le présent travail de Jan Goldstein sur la formation d'un «moi bourgeois» peut contribuer à l'interrogation sur l'existence ou non d'une conscience de classe bourgeoise dans la période post-révolutionnaire. 